\title{
A Tale of Three Galaxies: A "Clumpy" View of the Spectroscopically Anomalous Galaxies IRAS F10398+1455, IRAS F21013-0739 and SDSS J0808+3948
}

\author{
Yanxia Xie ${ }^{\mathrm{a}, \mathrm{b}, \mathrm{c}}$, Robert Nikutta ${ }^{\mathrm{d}}$, Lei Hao ${ }^{\mathrm{a}}$, Aigen $\mathrm{Li}^{\mathrm{b}}$ \\ ${ }^{a}$ Shanghai Astronomical Observatory, Chinese Academy of Sciences, 80 Nandan Road, \\ Shanghai 200030, China \\ ${ }^{b}$ Department of Physics and Astronomy, University of Missouri, Columbia, MO 65211, \\ USA \\ ${ }^{c}$ Kavli Institute for Astronomy and Astrophysics, Peking University, Beijing 100871, \\ China \\ ${ }^{d}$ Instituto de Astrofisica, Facultad de Fisica, Pontificia Universidad Católica de Chile, \\ 306, Santiago 22, Chile
}

\begin{abstract}
We investigate the dust properties in three spectroscopically anomalous galaxies (IRAS F10398+1455, IRAS F21013-0739 and SDSS J0808+3948) in terms of the 'CLUMPY' model for active galactic nuclei (AGNs). Their Spitzer/IRS spectra are characterized by a steep $\sim 5-8 \mu \mathrm{m}$ emission continuum, strong emission bands from polycyclic aromatic hydrocarbon (PAH) molecules, and prominent silicate emission. The steep $\sim 5-8 \mu \mathrm{m}$ emission continuum and strong PAH emission features suggest the dominance of starbursts, while the silicate emission is indicative of significant heating from AGNs. We find that the 'CLUMPY' model is generally successful in explaining the overall dust infrared emission, although it appears to emit too flat a $\sim 5-8 \mu$ m continuum to be consistent with that observed in IRAS F10398+1455 and IRAS F210130739. The flat $\sim 5-8 \mu \mathrm{m}$ continuum calculated from the 'CLUMPY' model could arise from the adopted specific silicate dust opacity. Future models
\end{abstract}

\footnotetext{
${ }^{*}$ Corresponding authors.

Email addresses: xieyx@shao.ac.cn;xieya@missouri.edu (Yanxia Xie), robert.nikutta@gmail.com (Robert Nikutta), haol@shao.ac.cn (Lei Hao), lia@missouri.edu (Aigen Li)
}

Preprint submitted to Planetary and Space Science

December 17, 2015

(C) 2015. This manuscript version is made available under the Elsevier user license http://www.elsevier.com/open-access/userlicense/1.0/ 
with a variety of dust species incorporated in the 'CLUMPY' radiation transfer regime are needed for a thorough understanding of the dust properties of these spectroscopically anomalous galaxies.

Keywords: Infrared, Extinction, Dust, Model

\section{Introduction}

Dust is a ubiquitous feature of the cosmos. It is present in a wide variety of astrophysical systems, including active galactic nuclei (AGNs) and starburst galaxies. Amorphous silicates and some form of carbonaceous materials (e.g., graphite, amorphous carbon, and hydrogenated amorphous carbon) are thought to be the two dominant cosmic dust species. Silicate dust reveals its presence in AGNs and starbursts through the 9.7 and $18 \mu \mathrm{m}$ spectral features which respectively arise from the $\mathrm{Si}-\mathrm{O}$ stretching and $\mathrm{O}-\mathrm{Si}-\mathrm{O}$ bending vibrational modes (Henning 2010).

In AGNs, some dust is hot enough to generate a strong, flat emission continuum in the 5-8 $\mu \mathrm{m}$ wavelength range, making it an effective diagnostic tool to distinguish AGN-dominated galaxies from starburst-dominated galaxies (e.g., see Laurent et al. 2000, Nardini et al. 2008). In addition to silicate dust, polycyclic aromatic hydrocarbon (PAH) molecules also display rich emission bands in the mid-IR (Tielens 2008). These features are usually very weak or absent in AGNs because PAHs are believed to have been destroyed by the harsh radiation field of AGNs (but also see Alonso-Herrero et al. 2014). The $3.4 \mu \mathrm{m} \mathrm{C-H}$ stretching absorption feature is also detected in AGNs which indicates the presence of aliphatic, chain-like hydrocarbon dust in AGNs (e.g., see Imanishi et al. 1997, Mason et al. 2004).

Compared to AGNs, starburst galaxies commonly display a low, red 5$8 \mu \mathrm{m}$ emission continuum, strong PAH emission features and silicate absorption features (e.g., Brandl et al. 2006, Hao et al. 2007, Smith et al. 2007). Starbursts lack the $3.4 \mu \mathrm{m}$ absorption feature. This may be related to the fact that starbursts, particularly in their nuclear regions, are often heavily obscured by dust and ice. In the Milky Way, the $3.4 \mu \mathrm{m}$ absorption feature is seen in the diffuse interstellar medium (ISM), but not in dense clouds where the $3.1 \mu \mathrm{m} \mathrm{H} \mathrm{H}_{2} \mathrm{O}$ ice absorption feature is strong (see Pendleton \& Allamandola 2002). Starbursts often exhibit strong $\mathrm{H}_{2} \mathrm{O}$ ice absorption at 3.1 and $6.0 \mu \mathrm{m}$ (e.g., see Spoon et al. 2004), while these ice features are not seen in AGNs where it is too hot for ice to survive against sublimation (see Li 2007). 
Very recently, Xie et al. (2014, hereafter paper I) studied the $\sim 5-40 \mu \mathrm{m}$ infrared (IR) spectra of IRAS F10398+1455, IRAS F21013-0739, and SDSS J0808+3948 obtained with the Infrared Spectrograph (IRS) on board the Spitzer Space Telescope (Houck et al. 2004). They found that the IR spectra of these galaxies are anomalous. Spectroscopically, on one hand they resemble that of AGNs in the sense that the silicate features in these galaxies are seen in emission; however, they also exhibit strong PAH emission features which are absent in AGNs. On the other hand, they are like starbursts in the sense that the $\sim 5-8 \mu \mathrm{m}$ emission continua of both these galaxies and starbursts all steeply rise with wavelength $\lambda$ and they all show strong PAH emission features. However, the silicate features seen in emission in these galaxies are often seen in absorption in starbursts. Furthermore, the steep $\sim 5-8 \mu \mathrm{m}$ emission continuum seen in these galaxies is much flatter in AGNs (see Figure 1 of Paper I). Let $d \ln F_{\nu} / d \ln \lambda$ be the slope of the $\sim 5-8 \mu \mathrm{m}$ emission continuum, where $F_{\nu}$ is the observed flux at frequency $\nu, \lambda=c / \nu$ is wavelength, and $c$ is the speed of light. On average, $d \ln F_{\nu} / d \ln \lambda \approx 0.8$ for AGNs, while for IRAS F10398+1455, IRAS F21013-0739, and SDSS J0808+3948, $d \ln F_{\nu} / d \ln \lambda \approx 4.1,4.2$, and 4.6, respectively. A detailed description of the spectral properties and a comparison of the Spitzer/IRS spectra of these three galaxies with that of typical starubursts and quasars can be found in Paper I.

Based on an extensive exploration of the multi-wavelength observational data, Y. Xie et al. (2016, in preparation) suggest that there might be a young/weak AGNs present in these three galaxies. The focus of this paper is to examine if the dust distribution in AGNs torus and/or the viewing angle plays a role in generating such an anomalous mid-IR spectral energy distribution (SED) in these three galaxies. This paper is organized as follows. We describe the data in $\S 2$. In $\S 3$ we describe the 'CLUMPY' dust model. The modelfitting to the observed IR emission is presented in $\S 4$. In $\S 5$ we discuss our results. The major conclusions are summarized in $\S 6$. Throughout the paper, we assume a cosmological model with $H_{0}=70 h_{70} \mathrm{~km} \mathrm{~s}^{-1} \mathrm{Mpc}^{-1}, \Omega_{m}=0.3$ and $\Omega_{\wedge}=0.7$. The $\mathrm{L}_{\odot}$ represents solar luminosity of $3.826 \times 10^{33} \mathrm{ergs} \mathrm{s}^{-1}$.

\section{Observations and Data}

These three galaxies were found when cross-matching the Sloan Digital Sky Survey (SDSS) and Spitzer/IRS low resolution spectra (L. Hao et al. 2016, in preparation). The Spitzer/IRS spectra of these galaxies are taken 
from the Cornell AtlaS of Spitzer/IRS Sources (CASSIS) which includes $\sim 13,000$ low resolution spectra of $>11,000$ distinct sources observed in the standard staring mode and provides publishable quality spectra (Lebouteiller et al. 2011). We tabulate their basic properties in Table 1.

Table 1: Basic Parameters for the Three Spectroscopically Anomalous Galaxies SDSS J0808+3948 IRAS F10398+1455 and IRAS F21013-0739

\begin{tabular}{lccccc}
\hline \hline Sources & R.A. & DEC. & Redshift & Program ID & $\begin{array}{c}L_{\mathrm{IR}} \\
\left(\mathrm{L}_{\odot}\right)\end{array}$ \\
\hline SDSS J0808+3948 & $08 \mathrm{~h} 08 \mathrm{~m} 44.27 \mathrm{~s}$ & $39 \mathrm{~d} 48 \mathrm{~m} 52.36 \mathrm{~s}$ & 0.091 & 40444 & $10^{10.84}$ \\
IRAS F10398 +1455 & $10 \mathrm{~h} 42 \mathrm{~m} 33.32 \mathrm{~s}$ & $+14 \mathrm{~d} 39 \mathrm{~m} 54.1 \mathrm{~s}$ & 0.099 & 40991 & $10^{10.50}$ \\
IRAS F21013-0739 & $21 \mathrm{~h} 03 \mathrm{~m} 58.75 \mathrm{~s}$ & $-07 \mathrm{~d} 28 \mathrm{~m} 02.5 \mathrm{~s}$ & 0.136 & 40444 & $10^{10.53}$ \\
\hline
\end{tabular}

While the $\sim 5-8 \mu \mathrm{m}$ continuum and the 9.7 and $18 \mu \mathrm{m}$ silicate emission features result from dust grains which, heated by the AGNs central engine, attain an equilibrium temperature (see Li 2007), the PAH emission features arise from PAH molecules which are stochastically heated by single stellar photons from their host galaxies (see Draine \& Li 2001). Due to the differences in their emission mechanisms, carriers, and emitting regions, to facilitate a detailed examination of the dust emission, we first remove the PAH emission features and the ionic emission lines from the Spitzer/IRS spectra of these galaxies. This requires an estimation of the dust emission continuum underneath the PAH and ionic emission lines. We approximate the continuum as a sum of starlight and blackbodies of different temperatures using a modified version of the PAHFIT software (Smith et al. 2007, see Paper I for details). The continuum determined in this way is hereafter referred to as "the PAHFIT continuum". We are mostly interested in the "residual" dust emission obtained by subtracting the PAH and ionic emission lines from the observed Spitzer/IRS spectra. For illustration, we show in Figure 1 the "residual" dust emission of SDSS J0808+3948, IRAS F10398+1455 and IRAS F21013-0739 obtained by subtracting the PAH and ionic emission lines determined from the PAHFIT approach.

\section{Dust Model}

The recipes of the 'CLUMPY' model were thoroughly described in Nekova et al. (2002, 2008a, 2008b). In this model the AGNs torus is populated by 

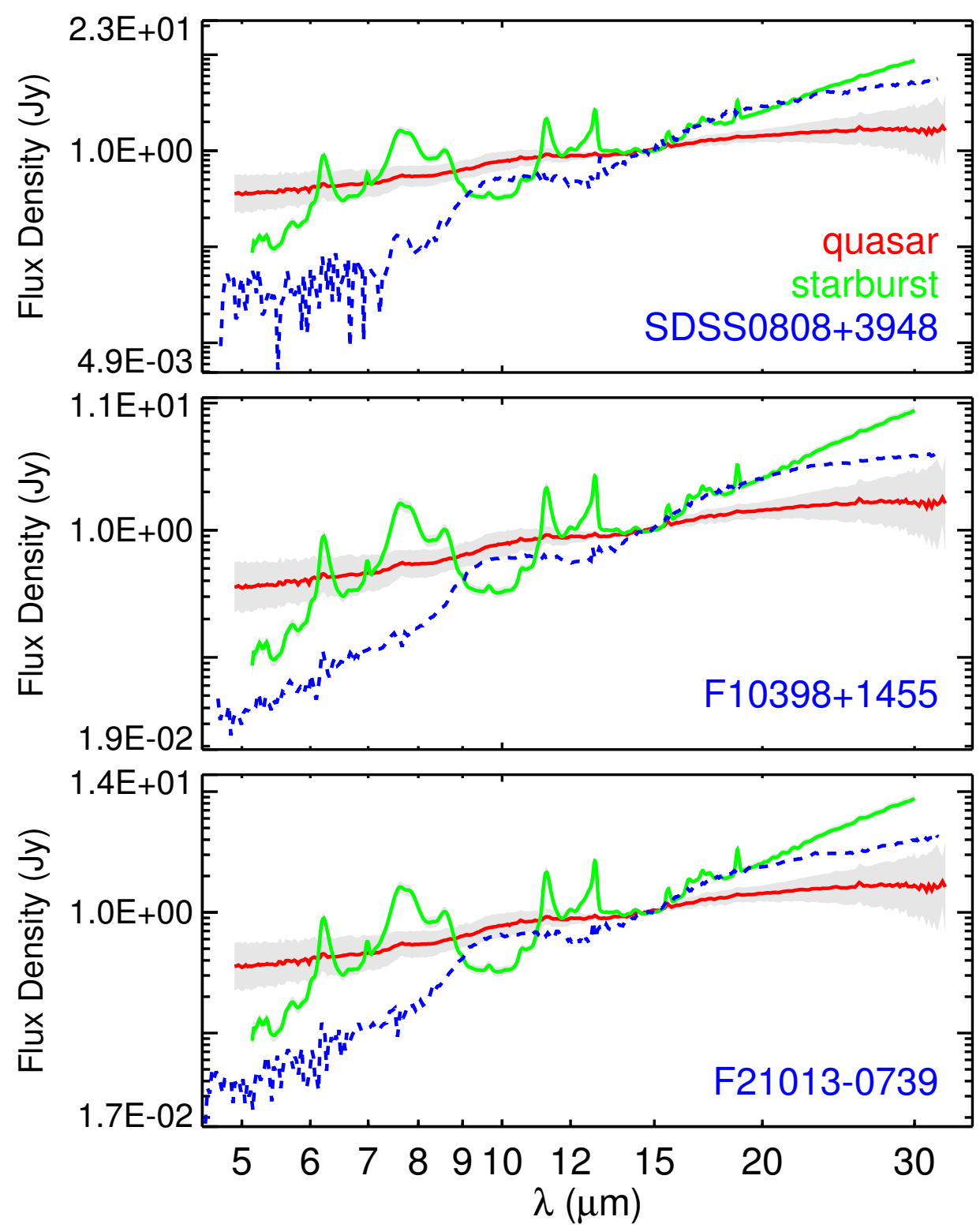

Figure 1: The "residual" dust emission of SDSS J0808+3948, IRAS F10398+1455 and IRAS F21013-0739 obtained by subtracting from the Spitzer/IRS spectrum the PAH and ionic emission lines determined from the PAHFIT approach (blue line ). In each panel, we also show the averaged IRS spectrum of quasar (red line)and starburst (green line) respectively for comparison. All spectra are nomalized at $\lambda=15 \mu \mathrm{m}$. 
dust clouds, with matter-free space between them. Radiation can propagate unabsorbed between the individually optically thick clouds. The visibility of the nucleus along the line-of-sight (LOS) to an external observer is probabilistic, and, in stark contrast to smooth-density torus models, is governed not only by the torus angular width and the viewing angle, but also by the clumpiness of the cloud distribution. An observer has a finite chance of seeing the unobscured nucleus even at edge-on views, if the photons generated in the central engine manage to avoid absorption by intervening torus clouds. Because clouds located at different position angles around the AGNs show different phases of their hot and cool faces, an external observer always registers emission from a mix of different dust temperatures, but from the same location around the AGNs. That these different dust temperatures co-exist at the same distance from the AGN is a hallmark characteristic of clumpiness. It has been confirmed observationally with the VLTI mid-IR observations (e.g., Jaffe et al. 2004, Raban et al. 2009).

The simplest possible general makeup of an axially symmetric clumpy cloud distribution requires six free parameters. The radial torus size $R_{o}=$ $Y R_{d}$ is a multiple of the scale-invariant dust sublimation radius $R_{d}$, which is set only by the AGNs luminosity. The multiplier $Y$ is a free parameter. The number of clouds is set by $N_{0}$, the mean number of clouds per radial ray in the equatorial plane. For radial rays $90-i$ degrees away from the equator this number falls off as a Gaussian with width $\sigma$ deg (measured from the equatorial plane). Our viewing angle $i$ onto the system is measured from the torus axis. The local cloud number density falls off radially as $1 / r^{q}$, with the power-law index $q$. Finally, the only property of a single cloud (in this minimal model) is its optical depth $\tau_{V}$ in the visual band. The dust grain size is assumed to follow a power-law distribution for each dust species.

\section{Results}

In this section we fit the PAH- and gas-lines subtracted residual SEDs of these three galaxies derived from $\S 2$ utilizing the 'CLUMPY' model presented in $\S 3$. We then find the best-fit model parameters and their marginalized posteriors using the Bayesian Markov-Chain Monte Carlo (MCMC) SED fitting code developed by Nikutta et al. (2012). It performs a multi-dimensional interpolation of the model SEDs at the requested parameter values and wavelengths, and allows estimation of model parameters including their significance. As priors, we assume uniform probability density distributions for all 
parameters, in light of their unknown true properties. During the fitting, the error bars for F10398+1455 and F21013-0739 are increased by a factor of 3 and 5 respectively to get the fitting converage in certain resolution.

We illustrate the results in Figure 2. From the best fitting model, it is apparent that the torus-only model can explain the SDSS J0808+3948 SED except the model $\mathrm{Si}-\mathrm{O}$ stretching feature of silicate dust peaks at $\sim 10.0 \mu \mathrm{m}$ while the observed silicate feature peaks at $\sim 9.7 \mu \mathrm{m}$. For F10398+1455 and F21013-0739, the model predicts too flat a $\sim 5-8 \mu \mathrm{m}$ continuum to be consistent with observed. In addition, like SDSS J0808+3948 the modelcalculated silicate emission feature for F10398+1455 and F21013-0739 also peaks at a wavelength longer than observed. These mismatches cannot be overcomed by adjusting the cloud distribution and viewing angle.

\section{Discussion}

We have shown that, no matter how we adjust the torus parameters, the dust IR emission SED calculated from the 'CLUMPY' model is too high in the $\sim 5-8 \mu \mathrm{m}$ wavelength range compared with the steep continuum of F10398+1455 and F21013-0739. The peak wavelength of the model silicate emission feature is at too long a wavelength for all three sources.

One possible reason for the mismatches may lie in the assumption of standard interstellar dust properties: the 'CLUMPY' model assumes a mass mixture of $53 \%$ silicate dust and $47 \%$ graphitic dust, with the optical constants of silicate and graphite respectively taken from Ossenkopf et al. (1992) and Draine (2003). We note that, among those AGNs exhibiting the $9.7 \mu \mathrm{m}$ (and $18 \mu \mathrm{m}$ ) silicate emission, a vast majority of them have their silicate emission peaking at a longer wavelength compared to that of the diffuse interstellar medium (e.g., Hao et al. 2005, Siebenmorgen et al. 2005, Sturm et al. 2005, Li et al. 2008, Smith et al. 2010, Shi et al. 2014). Nikutta et al. (2009) argued that the 'CLUMPY' model with the Ossenkopf et al. (1992) silicate can explain the majority of the AGNs of which the peak wavelengths of the silicate emission features are longward-shifted. We note that this is partly due to the fact that the opacity profile of the Ossenkopf et al. (1992) silicate actually has a longer peak position of $\sim 10.0 \mu \mathrm{m}$ compared to that of the interstellar silicate profile which peaks at $\sim 9.7 \mu \mathrm{m}$ (Kemper et al. 2004). However, for these three galaxies of interest in this paper, their silicate emission features all peak at $\sim 9.7 \mu \mathrm{m}$ and are comparable to that of the interstellar silicate. Therefore, it is unavoidable for the 'CLUMPY' model which is based on the 


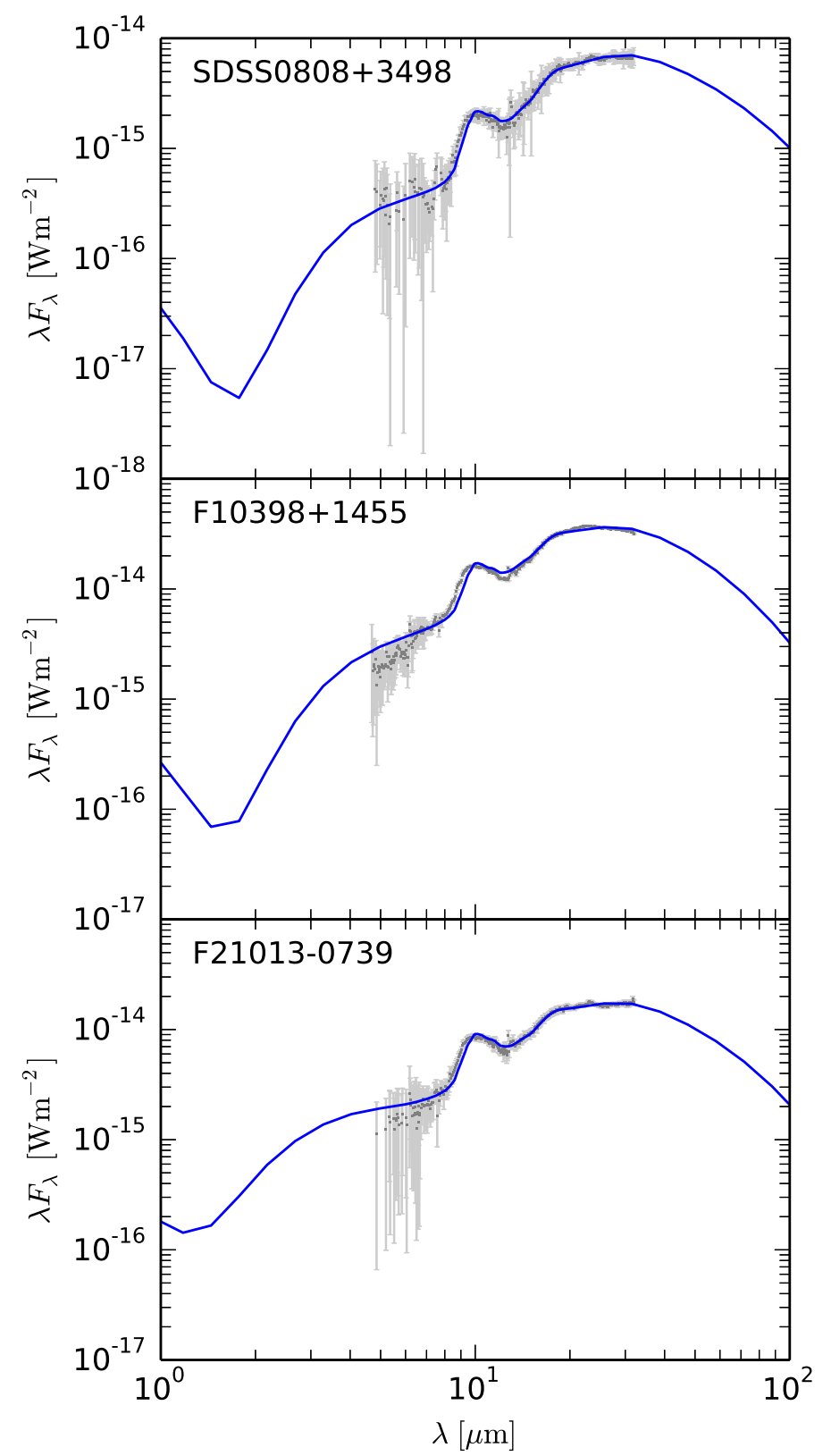

Figure 2: AGNs 'CLUMPY' model fitting to the PAH- and gas-lines subtracted residual spectra of SDSS J0808+3948, IRAS F10398+1455 and IRAS F21013-0739 obtained in $§ 2$. In each panel, the dark gray dots plot the observed data, the $1 \sigma$ error of each data point is denoted in gray lines. The solid blue line shows the best fit derived from the 'CLUMPY' model. For F10398+1455 and F21013-0739, the error bars are increased by a factor of 3 and 5 respectively to get the fitting converage in certain resolution. 
Ossenkopf et al. (1992) silicate to predict too long a peak wavelength for the silicate emission feature. Also, the mismatch to the steep $\sim 5-8 \mu \mathrm{m}$ continuum of F10398+1455 and F21013-0739 may be caused by the fixed mass ratio of silicate to graphite of $m_{\text {sil }} / m_{\text {gra }}=1.13$. A higher $m_{\text {sil }} / m_{\text {gra }}$ ratio may lead to a steep $\sim 5-8 \mu \mathrm{m}$ continuum. In Paper I, we have qualitatively discussed the possible dust properties that may account for the steep $\sim 5-$ $8 \mu \mathrm{m}$ continuum while exhibiting the prominent silicate emission feature in terms of dust composition, size and temperature. Based on the dust opacity characteristics presented in Figure 4 of Paper I, we proposed that sub- $\mu$ msized, iron-poor silicate as well as the deficit of carbon dust could probably be responsible for the anomalous SEDs observed in these three galaxies.

In Xie et al. (2015, hereafter paper II) such dust properties have been explored in terms of a simple optically thin model consisting of a mixture of warm and cold silicate dust, and warm and cold carbon dust. It was found that models consisting of "astronomical" silicate, amorphous olivine, or pyroxene, combined with amorphous carbon or graphite, are all capable of successfully fitting the observed IR emission. The dust temperature is the primary cause in regulating the steep $\sim 5-8 \mu \mathrm{m}$ continuum and silicate emission, insensitive to the exact silicate or carbon dust mineralogy. More specifically, the temperature of the $\sim 5-8 \mu \mathrm{m}$ continuum emitter (which is essentially carbon dust) of these galaxies is $\sim 250-400 \mathrm{~K}$, much lower than that of typical quasars which is $\sim 640 \mathrm{~K}$. The $9.7 \mu \mathrm{m}$ emission feature constrains the silicate dust size to not exceed $\sim 1.0 \mu \mathrm{m}$ (i.e., $a \lesssim 1.0 \mu \mathrm{m}$ ). The mass ratio of the warm carbon dust to the warm silicate dust ranges from $\sim 0.2$ to $\sim 2.0$, with a mean ratio of $\sim 0.98$. Similarly, the mass ratio of the cold carbon dust to the cold silicate dust ranges from $\sim 0.34$ to $\sim 2.0$, with a mean ratio of $\sim 1.5$. The total dust mass is dominated by the cold components, with the warm components only accounting for $<0.15 \%$ of the total dust mass.

To more effectively explore the dust properties of these three galaxies, one need to incorporate these different dust compositions and mass ratios into the 'CLUMPY' radiation transfer model. In Nikutta et al. (2016, in preparation), we will elaborately investigate these dust characteristics with the 'CLUMPY' model. 


\section{Summary}

We have modeled the Spitzer/IRS spectra of three spectroscopically anomalous galaxies (IRAS F10398+1455, IRAS F21013-0739 and SDSS J0808+3948) utilizing the AGNs 'CLUMPY' torus model. The IR spectral characteristics of these galaxies are unique in the sense that they show silicate emission which is characteristic of AGNs while they also show a steep $\sim 5-8 \mu$ m emission continuum and strong PAH emission features which are characteristic of starburst galaxies. In contrast, AGNs exhibit a flat emission continuum at $\sim 5-8 \mu \mathrm{m}$ and lack the PAH emission features. The 9.7 and $18 \mu \mathrm{m}$ silicate features seen in emission in these three galaxies are seen in absorption in starbursts.

We have investigated whether their anomalous SEDs could be explained by adjusting the dust cloud distribution and the viewing angle toward the AGNs torus. It is found that the 'CLUMPY' model is generally successful in explaining the overall dust IR emission, although it appears to emit too flat a $\sim 5-8 \mu$ m continuum to be consistent with that observed in IRAS F10398+1455 and IRAS F21013-0739. Also, the peak wavelength of the model silicate emission feature is too long compared with that observed in all three sources. We argue that the problem of the model-predicted longward-shifted silicate emission peak could be solved by incorporating silicate species other than the Ossenkopf et al. (1992) silicate, and the problem of the model-predicted flat $\sim 5-8 \mu \mathrm{m}$ continuum could be solved by incorporating iron-poor silicate dust and/or a higher silicate-to-graphite mass ratio than that currently adopted in the 'CLUMPY' model.

\section{Acknowledgements}

LH and XYX are partially supported by the 973 Program of China (2013CB834905, 2009CB824800), the Strategic Priority Research Program "The Emergence of Cosmological Structures" of Chinese Academy of Sciences (XDB09000000), the Shanghai Pujiang Talents Program (10pj1411800)

and NSFC 11073040, 11173019. AL and XYX are supported in part by NSF AST-1311804 and NASA NNX14AF68G. RN acknowledges support by FONDECYT grant No. 3140436. The Cornell Atlas of Spitzer/IRS Sources (CASSIS) is a product of the Infrared Science Center at Cornell University, supported by NASA and JPL. 
Allamandola, L.J., Tielens, A.G.G.M., Barker, J.R., 1985. Polycyclic Aromatic Hydrocarbons and the Unidentified Infrared Emission Bands - Auto Exhaust along the Milky Way. Astrophys. J. Lett. 290, L25-L28. doi: $10.1086 / 184435$

Alonso-Herrero, A., Ramos Almeida, C., Esquej, P., et al. 2014. Nuclear $11.3 \mu \mathrm{m}$ PAH emission in local active galactic nuclei. Mon. Not. R. Astron. Soc. 443, 2766-2782. doi: 10.1093/mnras/stu1293

Brandl, B. R., Bernard-Salas, J., Spoon, H. W. W., et al. 2006. The MidInfrared Properties of Starburst Galaxies from Spitzer-IRS Spectroscopy. Astrophys. J. 653, 1129-1144. doi: 10.1086/508849

Draine, B.T., 2003. Interstellar Dust Grains. Annu. Rev. Astron. Astrophys. J. 41, 241-289. doi: 10.1146/annurev.astro.41.011802.094840

Draine, B. T., \& Li, A. 2001. Infrared Emission from Interstellar Dust. I. Stochastic Heating of Small Grains. Astrophys. J. 551, 807-824. doi: $10.1086 / 320227$

Hao, L., Spoon, H. W. W., Sloan, G. C., et al. 2005. The Detection of Silicate Emission from Quasars at 10 and 18 Microns. Astrophys. J. Lett. 625, L75-L78. doi: 10.1086/431227

Hao, L., Weedman, D. W., Spoon, H. W. W., et al. 2007. The Distribution of Silicate Strength in Spitzer Spectra of AGNs and ULIRGs. Astrophys. J. Lett. 655, L77-L80. doi: 10.1086/511973

Hao, L., Lyu, J.W., Xie, Y., 2016. in preparation.

Henning, T. 2010. Cosmic Silicates. Annu. Rev. Astron. Astrophys. J. 48, 21-46. doi: 10.1146/annurev-astro-081309-130815

Houck, J. R., Roellig, T. L., van Cleve, J., et al. 2004. The Infrared Spectrograph (IRS) on the Spitzer Space Telescope. Astrophys. J. Suppl. Ser. 154, 18-24. doi: 10.1086/423134

Imanishi, M., Terada, H., Sugiyama, K., et al. 1997. Spectroscopic Study of NGC 1068 in the 3 micron Band. Publ. Astron. Soc. Japan. 49, 69-73. doi: $10.1093 / \mathrm{pasj} / 49.1 .69$ 
Jaffe, W., Meisenheimer, K., Röttgering, H. J. A., et al. 2004. The central dusty torus in the active nucleus of NGC 1068. Nature. 429, 47-49. doi: 10.1038 /nature02531

Kemper, F., Vriend, W. J., \& Tielens, A. G. G. M. 2004. The Absence of Crystalline Silicates in the Diffuse Interstellar Medium. Astrophys. J. 609, 826-837. doi: 10.1086/421339

Laurent, O., Mirabel, I. F., Charmandaris, V., et al. 2000. Mid-infrared diagnostics to distinguish AGNs from starbursts. Astron. Astrophys. 359, 887-899.

Lebouteiller, V., Barry, D. J., Spoon, H. W. W., et al. 2011. CASSIS: The Cornell Atlas of Spitzer/Infrared Spectrograph Sources. Astrophys. J. Suppl. Ser. 196, 8-20. doi: 10.1088/0067-0049/196/1/8

Li, A. 2007, In The Central Engine of Active Galactic Nuclei (ASP Conf. Ser. 373), ed. L. C. Ho \& J.-M. Wang (San Francisco, CA: ASP), 561-572

Li, M. P., Shi, Q. J., \& Li, A. 2008. On the anomalous silicate emission features of active galactic nuclei: a possible interpretation based on porous dust. Mon. Not. R. Astron. Soc. 391, L49-L53. doi: 10.1111/j.17453933.2008.00553.x

Mason, R. E., Wright, G., Pendleton, Y., \& Adamson, A. 2004. Hydrocarbon Dust Absorption in Seyfert Galaxies and Ultraluminous Infrared Galaxies. Astrophys. J. 613, 770-780. doi: 10.1086/423316

Nardini, E., Risaliti, G., Salvati, M., et al. 2008. Spectral decomposition of starbursts and active galactic nuclei in 5-8 $\mu \mathrm{m}$ Spitzer-IRS spectra of local ultraluminous infrared galaxies. Mon. Not. R. Astron. Soc. 385, L130-L134. doi: $10.1111 / \mathrm{j} .1745-3933.2008 .00450 . \mathrm{x}$

Nikutta, R., Elitzur, M., \& Lacy, M. 2009. On the $10 \mu \mathrm{m}$ Silicate Feature in Active Galactic Nuclei. Astrophys. J. 707, 1550-1559. doi: 10.1088/0004$637 \mathrm{X} / 707 / 2 / 1550$

Nikutta, R., \& Elitzur, M. 2012. Fundamental limits of parameter constraining power in torus modeling. Torus Workshop, 2012, 205

Nikutta, R., Xie, Y., Li, A., \& Hao, L., 2016. in preparation. 
Nenkova, M., Ivezić, Ž., \& Elitzur, M. 2002. Dust Emission from Active Galactic Nuclei. Astrophys. J. Lett. 570, L9-L12. doi: 10.1086/340857

Nenkova, M., Sirocky, M. M., Ivezić, Ž., \& Elitzur, M. 2008a. AGN Dusty Tori. I. Handling of Clumpy Media. Astrophys. J. 685, 147-159. doi: $10.1086 / 590482$

Nenkova, M., Sirocky, M. M., Nikutta, R., Ivezić, Ž., \& Elitzur, M. 2008b. AGN Dusty Tori. II. Observational Implications of Clumpiness. Astrophys. J. 685, 160-180. doi: 10.1086/590483

Ossenkopf, V., Henning, T., \& Mathis, J. S. 1992. Constraints on cosmic silicates. Astron. Astrophys. 261, 567-578.

Pendleton, Y.J., Allamandola, L.J., 2002. The Organic Refractory Material in the Diffuse Interstellar Medium: Mid-Infrared Spectroscopic Constraints. Astrophys. J. Suppl. Ser. 138, 75-98. doi: 10.1086/322999

Raban, D., Jaffe, W., Röttgering, H., Meisenheimer, K., \& Tristram, K. R. W. 2009. Resolving the obscuring torus in NGC 1068 with the power of infrared interferometry: revealing the inner funnel of dust. Mon. Not. R. Astron. Soc. 394, 1325-1337. doi: 10.1111/j.1365-2966.2009.14439.x

Shi, Y., Rieke, G. H., Ogle, P. M., Su, K. Y. L., \& Balog, Z. 2014. Infrared Spectra and Photometry Of Complete Samples of Palomar-Green and Two Micron All Sky Survey Quasars. Astrophys. J. Suppl. Ser. 214, 23-39. doi: $10.1088 / 0067-0049 / 214 / 2 / 23$

Siebenmorgen, R., Haas, M., Krügel, E., \& Schulz, B. 2005. Discovery of $10 \mu \mathrm{m}$ silicate emission in quasars. Evidence of the AGN unification scheme. Astron. Astrophys. 436, L5-L8. doi: 10.1051/0004-6361:200500109

Smith, J. D. T., Draine, B. T., Dale, D. A., et al. 2007. The MidInfrared Spectrum of Star-forming Galaxies: Global Properties of Polycyclic Aromatic Hydrocarbon Emission. Astrophys. J. 656, 770-791. doi: $10.1086 / 510549$

Smith, H. A., Li, A., Li, M. P., et al. 2010. Anomalous Silicate Dust Emission in the Type 1 Liner Nucleus of M81. Astrophys. J. 716, 490-503. doi: 10.1088/0004-637X/716/1/490 
Spoon, H. W. W., Armus, L., Cami, J., et al. 2004. Fire and Ice: Spitzer Infrared Spectrograph (IRS) Mid-Infrared Spectroscopy of IRAS F001837111. Astrophys. J. Suppl. Ser. 154, 184-187. doi: 10.1086/422813

Sturm, E., Schweitzer, M., Lutz, D., et al. 2005. Silicate Emissions in Active Galaxies: From LINERs to QSOs. Astrophys. J. Lett. 629, L21-L23. doi: $10.1086 / 444359$

Tielens, A. G. G. M. 2008. Interstellar Polycyclic Aromatic Hydrocarbon Molecules. Annu. Rev. Astron. Astrophys. J. 46, 289-337. doi: 10.1146/annurev.astro.46.060407.145211

Xie, Y., Hao, L., \& Li, A. 2014. A Tale of Three Galaxies: Anomalous Dust Properties in IRAS F10398+1455, IRAS F21013-0739, and SDSS J0808+3948. Astrophys. J. Lett. 794, L19-L24. doi: 10.1088/2041$8205 / 794 / 2 /$ L19

Xie, Y., Li, A., Hao, L., \& Nikutta, R. 2015. A Tale of Three Galaxies: Deciphering the Infrared Emission of the Spectroscopically Anomalous Galaxies IRAS F10398+1455, IRAS F21013-0739, and SDSS J0808+3948. Astrophys. J. 808, 145-157. doi: 10.1088/0004-637X/808/2/145

Xie, Y., Hao, L., Charmandaris, V., Diaz-Santos, T., Nikutta, R., 2016. in preparation. 